OPEN ACCESS

Edited by:

Nader Hanna

University of Maryland, Baltimore,

United States

Reviewed by:

Fuyuhiko Motoi,

Yamagata University, Japan

Stefano Francesco Crinò,

University of Verona, Italy

${ }^{*}$ Correspondence:

Xu Che

yixuetg@foxmail.com

Specialty section:

This article was submitted to Gastrointestinal Cancers: Hepato

Pancreatic Biliary Cancers, a section of the journal

Frontiers in Oncology

Received: 28 August 2021 Accepted: 18 October 2021 Published: 04 November 2021

Citation:

Zhang W, Xu L and Che X (2021)

Nomogram for Predicting the Prognoses of Patients With Pancreatic Head Cancer After

Pancreaticoduodenectomy: A Population-Based

Study on SEER Data.

Front. Oncol. 11:766071. doi: 10.3389/fonc.2021.766071

\section{Nomogram for Predicting the Prognoses of Patients With Pancreatic Head Cancer After Pancreaticoduodenectomy: A Population-Based Study on SEER Data}

\author{
Wei Zhang ${ }^{1,2}$, Lin $X u^{1}$ and $X u$ Che ${ }^{1,2 *}$
}

\begin{abstract}
1 Department of Hepatobiliary and Pancreatic Surgery, National Cancer Center/National Clinical Research Center for Cancerl Cancer Hospital \& Shenzhen Hospital, Chinese Academy of Medical Sciences and Peking Union Medical College, Shenzhen, China, ${ }^{2}$ Department of Pancreatic and Gastric Surgery, National Cancer Center/National Clinical Research Center for Cancer/Cancer Hospital, Chinese Academy of Medical Sciences and Peking Union Medical College, Beijing, China
\end{abstract}

Objective: In this study, we retrieved the data available in the Surveillance, Epidemiology, and End Results database to identify the prognostic factors for patients with pancreatic head cancer who had undergone pancreaticoduodenectomy and developed a prediction model for clinical reference.

Methods: We screened the data between 1973 and 2015. Propensity score matching (PSM) was used to control for the confounding factors. Kaplan-Meier (log-rank test) curves were used to compare the survival rates. A nomogram was established using multifactorial Cox regression.

Results: In total, 4099 patients were identified. Their median survival was 22 months, with $74.2 \%, 36.5 \%$, and $26.2 \%$ survival after 1,3 , and 5 years, respectively. The median cancerspecific survival was 24.0 months, with $71.1 \%, 32.6 \%$, and $21.9 \%$ survival after 1 , 3, and 5 years, respectively. The results of the Cox proportional risk regression showed that age, insurance status, gender, histological type, degree of tissue differentiation, $\mathrm{T}$ and $\mathrm{N}$ stages, tumor size, extent of regional lymph node dissection, and postoperative radiotherapy or chemotherapy are independent factors affecting prognosis. PSM was used twice to eliminate any bias from the unbalanced covariates in the raw data. After PSM, the patients who had received postoperative radiotherapy were found to have a better survival prognosis and disease-specific survival prognosis than those who had not received radiotherapy $[\mathrm{HR}=$ 0.809, 95\% Cl (0.731-0.894), $P<0.001$ and $\mathrm{HR}=0.814,95 \% \mathrm{Cl}(0.732-0.904), P<0.001$; respectively]. A similar result was observed for the patients who had received postoperative chemotherapy versus those who had not [HR $=0.703,95 \% \mathrm{Cl}(0.633-0.78), P<0.001$ and 
$\mathrm{HR}=0.736,95 \% \mathrm{Cl}(0.658-0.822), P<0.001$, for survival and disease-specific survival prognoses, respectively]. Finally, the $\beta$ coefficients of the Cox proportional risk regression were used to establish a nomogram.

Conclusion: Age, insurance status, gender, histological type, degree of differentiation, $T$ and $\mathrm{N}$ stages, tumor size, regional lymph node dissection, and postoperative radiotherapy or chemotherapy are factors affecting the prognosis in pancreatic head cancer after pancreaticoduodenectomy. Postoperative radiotherapy and chemotherapy can improve patient survival. These still need to be further validated in the future.

Keywords: pancreatic adenocarcinoma, pancreaticoduodenectomy, prognosis, predictive model, nomogram

\section{INTRODUCTION}

The new cases of pancreatic cancer in the United States in 2017 corresponded to $9 \%$ of all the cases of malignancies in the country. This cancer type is the ninth most common cancer and the fourth cause of cancer-related deaths in the United States, with an estimated 53,670 new cases and 43,090 deaths in 2017 (1). In China, there is an increasing trend in the incidence of pancreatic cancer, which has the 7 th highest mortality rate among all the malignancies (2). Since there is no specific method for early detection of pancreatic head cancer and pancreatic tumors progress rapidly, patients are often lost to surgery at the time of diagnosis. Released data from the Surveillance, Epidemiology, and End Results (SEER) database show that, of the 20,470 patients diagnosed with pancreatic head cancer between 2010 and 2015, only 37.6\% (7,688/20,470) had undergone surgery, with pancreaticoduodenectomy accounting for $78.5 \%(6,037 / 7,688)$ of all the surgical procedures. Pancreaticoduodenectomy, also known as the Whipple procedure, was first reported in 1935 by Whipple (3). This procedure is considered to be one of the most complex abdominal surgeries and characterized by high trauma and complication rates since it requires removal of numerous organs and reconstruction of the digestive tract, including the pancreas, biliary tract, and gastrointestinal tract. The median survival period of patients with pancreatic head cancer is less than two years after the surgery. In the face of such a complex procedure with extremely high postoperative complications, new methods that can effectively and accurately predict the postoperative survival rate should be identified and evaluated to establish an optimal customized treatment strategy.

The TNM staging guidelines developed by the American Joint Committee on Cancer (AJCC) are widely used in clinical practice to predict prognoses of patients with pancreatic cancer. This staging procedure is based on tumor size, extent of invasion, and lymph node and metastatic statuses. Although it is used to predict the post-surgery prognosis of patients with pancreatic cancer, its precision is often sub-optimal. There are also other potential factors affecting the prognosis, including postoperative radiotherapy or chemotherapy. Therefore, more accurate and specific prediction models than TNM staging are needed for prognostic analysis of patients with pancreatic head cancer who underwent radical pancreaticoduodenectomy.
Nomogram is a simple multivariate prediction model that incorporates multiple variables affecting prognosis to calculate the survival probability of an individual (4). Recently, disease-specific nomograms have been increasingly used for prognostic prediction in various malignancies (5-11). In addition, with the promotion and development of adjuvant therapy, treatment of pancreatic cancer has taken a multidisciplinary and comprehensive form, with surgery constituting the core. Therefore, this study aimed to develop and validate a nomogram with better applicability and higher predictive accuracy that can be used for individualized assessment of post-surgery survival of pancreatic head cancer patients undergoing pancreaticoduodenectomy.

\section{MATERIALS AND METHODS}

\section{Population and Inclusion Criteria}

This retrospective cohort study evaluated the survival of patients with pancreatic head cancer who had undergone pancreaticoduodenectomy. The patient data were derived from the database of SEER, a cancer surveillance research program established by the National Cancer Institute in 1973 and used by the National Institutes of Health to collect the cancer statistics in the U.S. The database currently has an ongoing collection of data related to cancer incidence, prevalence, and survival rate from 18 regional registries, tracking approximately $34.6 \%$ of the U.S. population. In this study, we evaluated the prognoses of patients in the SEER database who were diagnosed with primary pancreatic head cancer between 2010 and 2015. Our exclusion criteria were i) incomplete data or missing data with important variables, including histopathological information and type of surgery, and ii) patients with secondary pancreatic cancer.

The clinical data in the SEER database include age, race, sex, primary site, histological type, degree of tissue differentiation, $\mathrm{T}$ and $\mathrm{N}$ stages, tumor size, regional lymph node dissection, radiotherapy, chemotherapy, survival time, cut-off time, survival status, and cause of death. In the SEER database, the histological grades of the tumors include grade I (welldifferentiated), grade II (moderately differentiated), grade III (poorly differentiated), and grade IV (undifferentiated). Pancreatic cancer is defined using the criteria of International Classification of Diseases in Oncology, 3rd edition (ICD-O-3), 
code C23.9. The histological types include epithelial neoplasms (codes 8010-8049); adenomas and adenocarcinomas (codes 8140-8389); cystic, mucinous, and serous neoplasms (codes 8440-8499); ductal and lobular neoplasms (codes 8500-8549); and complex epithelial neoplasms (codes 8560-8579). We reviewed the SEER database based on the procedure record codes and the SEER Data Variable Dictionary to distinguish between the different procedure types. The item " 37 " in the SEER data "RX Summ-Surg Prim Site (1998+)" (NAACCR item 1290, code 37) corresponds to "Pancreatic Duodenectomy (Whipple)". Cause-specific survival (CSS) is defined in this article as the cause of death due to pancreatic cancer. The CSS corresponds to the record "Pancreas" in the variable "COD to site rec KM." It was calculated by the SEER database by using algorithms that extracted the cause of death from the death certificates to determine a single, disease-specific cause of death. Notably, in some cases, attribution of a single cause of death may be difficult and incorrect. For example, the cause of death may be attributed to the site of metastasis rather than the primary site.

\section{Statistical Analysis}

Categorical variables were presented as frequencies (\%), and $\chi 2$ or Fisher's exact test was used for comparative analysis. Continuous variables were presented as mean $\pm \mathrm{SD}$ or median [interquartile range (IQR)], and independent samples $t$-test or Mann-Whitney U-test was used for comparative analysis. Survival curves were plotted using the Kaplan-Meier method and compared using the log-rank test. The risk ratio (HR) was calculated using the Cox proportional risk model. The SEER data were extracted using SEER ${ }^{\star}$ Stat 8.3.5 in client-server mode. Statistical analyses were performed using SPSS Statistics 24.0 (IBM, Chicago, IL) and R software (https://www.r-project.org/) along with the optional packages of the software. Two-tailed $P$ values $<0.05$ were considered statistically significant.

\section{Propensity Score Matching (PSM)}

Another objective of this study was to assess the impact of postoperative radiotherapy or chemotherapy on survival. The uneven distribution of the patient characteristics in the SEER database population usually biases the conclusions. In this study, via PSM on the raw data, we controlled for covariates that would affect the treatment selection process. The covariates include identified prognostic factors and other factors or variables in the SEER data. The propensity score was considered to be the probability of a patient to receive postoperative radiotherapy or chemotherapy and estimated using a non-parsimonious logistic multiple regression model. A nearest-neighbor matching algorithm (ratio = 1: 1 without replacement) was used, and the caliper width was 0.05 SDs of the logit model used to calculate the scores.

\section{Multivariate Cox Regression Analysis and Nomogram Development and Validation}

A multifactorial regression analysis was performed using a Cox proportional risk model. The obtained variables were included in the model to calculate the effect of these factors on the survival benefit of patients after pancreaticoduodenectomy. The $\beta$ coefficients from the multifactorial Cox regression analysis were used to establish the nomogram. Calibration and receiver operating characteristic curves were used to validate the predictive performance of the model.

\section{RESULTS}

\section{Search Results and Characteristics of the Included Patients}

By applying the criteria stated above, this retrospective study initially identified that the SEER database had 7688 patients with stage M0 pancreatic head cancer who had undergone surgery between 2010 and 2015. However, we excluded 2,939 of these patients from this study because they had undergone procedures other than pancreaticoduodenectomy, such as mass enucleation and partial pancreatectomy. In addition, additional 650 patients were excluded because of unknown $\mathrm{T}$ or $\mathrm{N}$ stage, extent of lymph node dissection, pathological tissue, tissue differentiation, or tumor size. We finally identified 4099 patients. The flow chart of data acquisition and screening is shown in Figure 1. The demographic and clinicopathological characteristics of the patients obtained from the SEER database are shown in Table 1.

\section{Univariate and Multifactorial Cox Regression Analysis of Survival Prognosis}

Univariate Cox regression analysis showed that age, gender, insurance status, histological classification, degree of tissue differentiation, $\mathrm{T}$ and $\mathrm{N}$ stages, tumor size $(\mathrm{mm})$, and postoperative chemotherapy (Yes/No) or radiotherapy (Yes/ No) are factors that affect the prognosis, whereas race and extent of regional lymphadenectomy are not. Multifactorial Cox regression analysis showed that age, gender, insurance status, histological type, degree of tissue differentiation, $\mathrm{T}$ and $\mathrm{N}$ stages, tumor size $(\mathrm{mm})$, postoperative chemotherapy (Yes/ No) or radiotherapy (Yes/No), and extent of regional lymph node dissection are independent factors affecting the prognosis, whereas race is not (Table 2).

\section{PSM and Survival Analysis of the Patients Treated With Postoperative Radiotherapy or Chemotherapy}

Data from 4099 patients identified from the SEER database were used for analysis. Of these patients, 1236 underwent only surgery (S group), 38 underwent surgery and postoperative radiotherapy ( $+\mathrm{R}$ group), 1597 underwent surgery and postoperative chemotherapy ( $+\mathrm{C}$ group), 129 underwent preoperative radiotherapy, surgery, and postoperative chemotherapy $(\mathrm{R}+\mathrm{S}+\mathrm{C}$ group), and 1099 underwent surgery, postoperative radiotherapy, and postoperative chemotherapy $(\mathrm{S}+\mathrm{R}+\mathrm{C}$ group). Their postoperative median survivals were 16, 19, 23, 24, and 25 months, respectively, and the pancreatic-cancer-specific median 


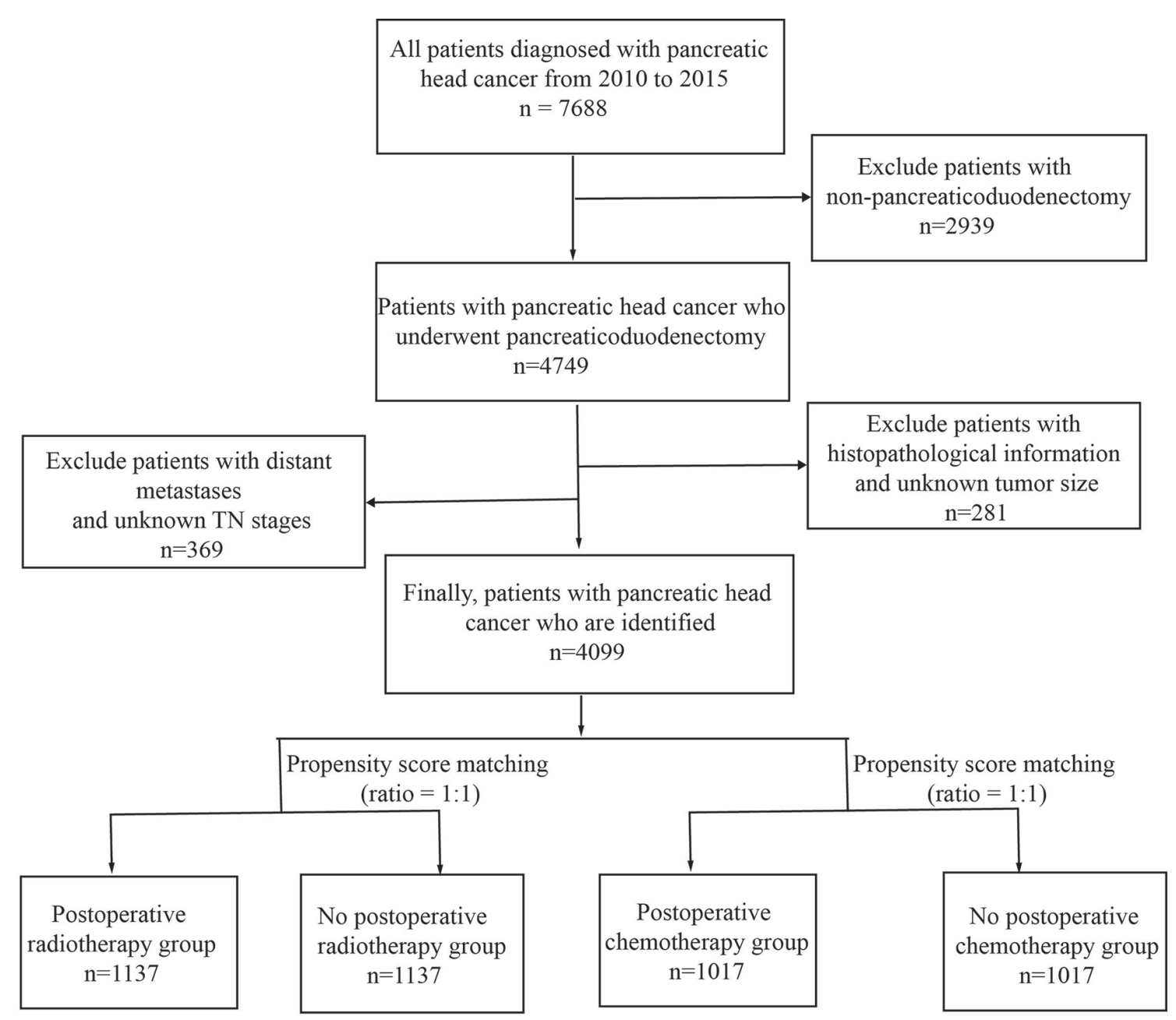

FIGURE 1 | Flow chart of the study-population screening.

survivals were $19,19,24,25,27$, and 24 months, respectively. Their all K-M survival curves are shown in Figure 2. The patients who received preoperative radiotherapy also received postoperative chemotherapy and were not significantly different from those who received only postoperative chemotherapy [Log Rank (Mantel-Cox) test $=0.177]$.

To eliminate the potential bias from preoperative radiotherapy, the 129 patients in the $\mathrm{R}+\mathrm{S}+\mathrm{C}$ group were removed, and the

TABLE 1 | Demographic and clinicopathological characteristics of the included population.

\section{Variable}

\section{Age (year)}

$\operatorname{Sex}(\mathrm{m} / \mathrm{f})$

Insurance (Medicaid/Insured/Uninsured)

Race (White/Black/Other)

Histology classification (ICD-O-3)

Grade (I/II/III/IV)

T stage (T1/T2/T3/T4)

$\mathrm{N}$ stage(N0/N1)

Scope Reg LN(None/1 3/4+)

Tumor size(mm)

Radiotherapy (Yes /No)

Chemotherapy (Yes /No)

\section{$\mathrm{N}=4099, \mathrm{n}(\%)$ or mean (SD)}

$65.91 \pm 10.74$

$2116,51.6 \% / 1983,48.4 \%$

409, 10\% / 3603, 87.9\% /87, 2.1\%

$3371,82.2 \% / 390,9.5 \% / 338,8.2 \%$

$42,1.0 \% / 2106,51.4 \% / 148,3.6 \% / 1771,43.2 \% / 32,0.8 \%$

632, 15.4\% / 2023, 49.4\%/1386, 33.8\% /58, 1.4\%

$248,6.1 \%$ / 459, $11.2 \%$ / 3237, $79.0 \%$ / 155, 3.8\%

$1324,32.3 \% / 2775,67.7 \%$

$53,1.3 \% / 122,3.0 \% / 3924,95.7 \%$

$32.71 \pm 14.09$

2833, $69.1 \% / 129,3.1 \% / 1137,27.7 \%$

$1274,31.1 \% / 2825,68.9 \%$

m, male; f, female; Scope Reg LN, Scope of regional lymphadenectomy ; ICD-O-3, The International Classification of Diseases for Oncology, Third Edition; SD, Standard deviation. 
TABLE 2 | Univariate and multivariate cox regression analyses of the prognoses of the patients with pancreatic head cancer who had undergone pancreaticoduodenectomy.

\begin{tabular}{|c|c|c|c|c|c|c|}
\hline \multirow[t]{2}{*}{ Variable } & \multicolumn{2}{|c|}{ Univariate analysis } & \multirow[t]{2}{*}{$p$} & \multicolumn{2}{|c|}{ Multivariate analysis } & \multirow[t]{2}{*}{$p$} \\
\hline & HR & $95 \% \mathrm{Cl}$ & & HR & $95 \% \mathrm{Cl}$ & \\
\hline Age (year) & 1.021 & $1.017 \sim 1.025$ & $<0.001$ & 1.017 & $1.013 \sim 1.021$ & $<0.001$ \\
\hline \multicolumn{7}{|l|}{ Sex } \\
\hline male & Reference & & & Reference & & \\
\hline female & 0.909 & $0.843 \sim 0.980$ & 0.012 & 0.89 & $0.825 \sim 0.960$ & 0.003 \\
\hline Insurance & & & 0.003 & & & $<0.001$ \\
\hline Medicaid & Reference & & & Reference & & \\
\hline Insured & 0.809 & $0.717 \sim 0.913$ & 0.001 & 0.735 & $0.650 \sim 0.832$ & $<0.001$ \\
\hline Uninsured & 0.782 & $0.584 \sim 1.047$ & 0.99 & 0.783 & $0.583 \sim 1.052$ & 0.104 \\
\hline Race (White/Black/Other) & & & 0.163 & & & 0.326 \\
\hline Histology classification (ICD-O-3) & & & $<0.001$ & & & 0.001 \\
\hline Epithelial neoplasms & Reference & & & Reference & & 0.296 \\
\hline Adenomas and adenocarcinomas & 1.018 & $0.700 \sim 1.480$ & 0.927 & 1.391 & $0.902 \sim 2.145$ & 0.135 \\
\hline Cystic, mucinous and serous & 0.731 & $0.475 \sim 1.126$ & 0.156 & 1.184 & $0.732 \sim 1.916$ & 0.49 \\
\hline Ductal and lobular neoplasms & 1.229 & $0.845 \sim 1.789$ & 0.281 & 1.583 & $1.024 \sim 2.447$ & 0.039 \\
\hline Complex epithelial neoplasms & 1.954 & $1.151 \sim 3.318$ & 0.013 & 1.934 & $1.090 \sim 3.430$ & 0.024 \\
\hline Grade & & & $<0.001$ & & & $<0.001$ \\
\hline Grade I (well differentiated) & Reference & & & Reference & & \\
\hline Grade II(moderately differentiated) & 1.979 & $1.737 \sim 2.253$ & $<0.001$ & 1.877 & $1.640 \sim 2.148$ & $<0.001$ \\
\hline Grade III (poorly differentiated) & 2.745 & $2.402 \sim 3.136$ & $<0.001$ & 2.515 & $2.186 \sim 2.893$ & $<0.001$ \\
\hline Grade IV (undifferentiated) & 2.391 & $1.727 \sim 3.309$ & $<0.001$ & 2.927 & $2.021 \sim 4.241$ & $<0.001$ \\
\hline T stage & & & $<0.001$ & & & $<0.001$ \\
\hline $\mathrm{T} 1$ & Reference & & & Reference & & \\
\hline T2 & 1.715 & $1.356 \sim 2.168$ & $<0.001$ & 1.296 & $1.016 \sim 1.653$ & 0.037 \\
\hline T3 & 2.582 & $2.102 \sim 3.171$ & $<0.001$ & 1.675 & $1.345 \sim 2.087$ & $<0.001$ \\
\hline T4 & 3.53 & $2.704 \sim 4.609$ & $<0.001$ & 2.411 & $1.811 \sim 3.210$ & $<0.001$ \\
\hline $\mathrm{N}$ stage & & & $<0.001$ & & & $<0.001$ \\
\hline NO & Reference & & & Reference & & \\
\hline N1 & 1.765 & $1.620 \sim 1.923$ & $<0.001$ & 1.669 & $1.524 \sim 1.829$ & $<0.001$ \\
\hline Scope Reg LN Sur & & & 0.557 & & & 0.002 \\
\hline None & Reference & & & Reference & & \\
\hline $1 \sim 3$ & 1.098 & $0.735 \sim 1.639$ & 0.649 & 1.042 & $0.695 \sim 1.562$ & 0.842 \\
\hline 4 or more & 0.976 & $0.693 \sim 1.376$ & 0.891 & 0.734 & $0.518 \sim 1.040$ & 0.082 \\
\hline Tumor size(mm) & 1.008 & $1.005 \sim 1.010$ & $<0.001$ & 1.004 & $1.001 \sim 1.007$ & 0.003 \\
\hline \multicolumn{7}{|l|}{ Chemotherapy (Yes/No) } \\
\hline No & Reference & & & Reference & & \\
\hline Yes & 0.773 & $0.713 \sim 0.838$ & $<0.001$ & 0.592 & $0.539 \sim 0.650$ & $<0.001$ \\
\hline Radiotherapy (Yes/No) & & & $<0.001$ & & & 0.011 \\
\hline None & Reference & & & Reference & & \\
\hline Preoperative radiotherapy & 0.8 & $0.643 \sim 0.995$ & 0.045 & 1.022 & $0.813 \sim 1.283$ & 0.855 \\
\hline Postoperative radiotherapy & 0.798 & $0.733 \sim 0.868$ & $<0.001$ & 0.867 & $0.788 \sim 0.954$ & 0.004 \\
\hline
\end{tabular}

m, male; f, female; Scope Reg LN, Scope of regional lymphadenectomy; ICD-O-3, The International Classification of Diseases for Oncology, Third Edition; PSM- Propensity score matching; Bold indicates a significant difference.

remaining patients were matched based on the propensity scores. Table 3 shows the baseline clinicopathological characteristics of the patients divided into two groups according to whether postoperative radiotherapy was administered. The two groups had unbalanced baseline characteristics of age, tumor size, insurance status, gender, histological type, degree of tissue differentiation, $\mathrm{T}$ and $\mathrm{N}$ stages, and postoperative chemotherapy (with/without); therefore, these factors were matched as covariates for a 1: 1 propensity score to obtain two balanced groups (Table 3). Survival and pancreatic-cancer-specific survival rates were found to be higher in the 1137 patients who had received postoperative radiotherapy than in the 1137 patients who had not. Their median postoperative survivals were 25 and 22 months, respectively [HR $=0.809,95 \%$ CI $(0.731-0.894), P<0.001$, and their pancreatic-cancer-specific survivals were 24 and 27 months, respectively $[\mathrm{HR}=0.814,95 \%$ CI $(0.732-0.904), P<$ 0.001] (Figure 3).
Table 4 shows the baseline clinical characteristics of the patients who had received postoperative chemotherapy versus those of the patients who had not. Patients who had received postoperative chemotherapy were imbalanced with those who had not in terms of age at diagnosis, tumor size, insurance status, histological type, degree of tissue differentiation, $\mathrm{T}$ and $\mathrm{N}$ stages, extent of regional lymph node clearance, and postoperative radiotherapy (Yes/No). Therefore, these factors were included as covariates in the model for 1: 1 PSM to obtain two balanced groups of patients (Table 4). Then, further analysis showed that the postoperative survival and pancreatic-cancer-specific survival rates of the 1017 patients who had received postoperative chemotherapy were higher than those of the 1017 patients who had not; the median survival rates were 24 and 14 months, respectively [HR $=0.703,95 \% \mathrm{CI}(0.633-0.78), P$ $<0.001]$, and the pancreatic-cancer-specific survival rates were 


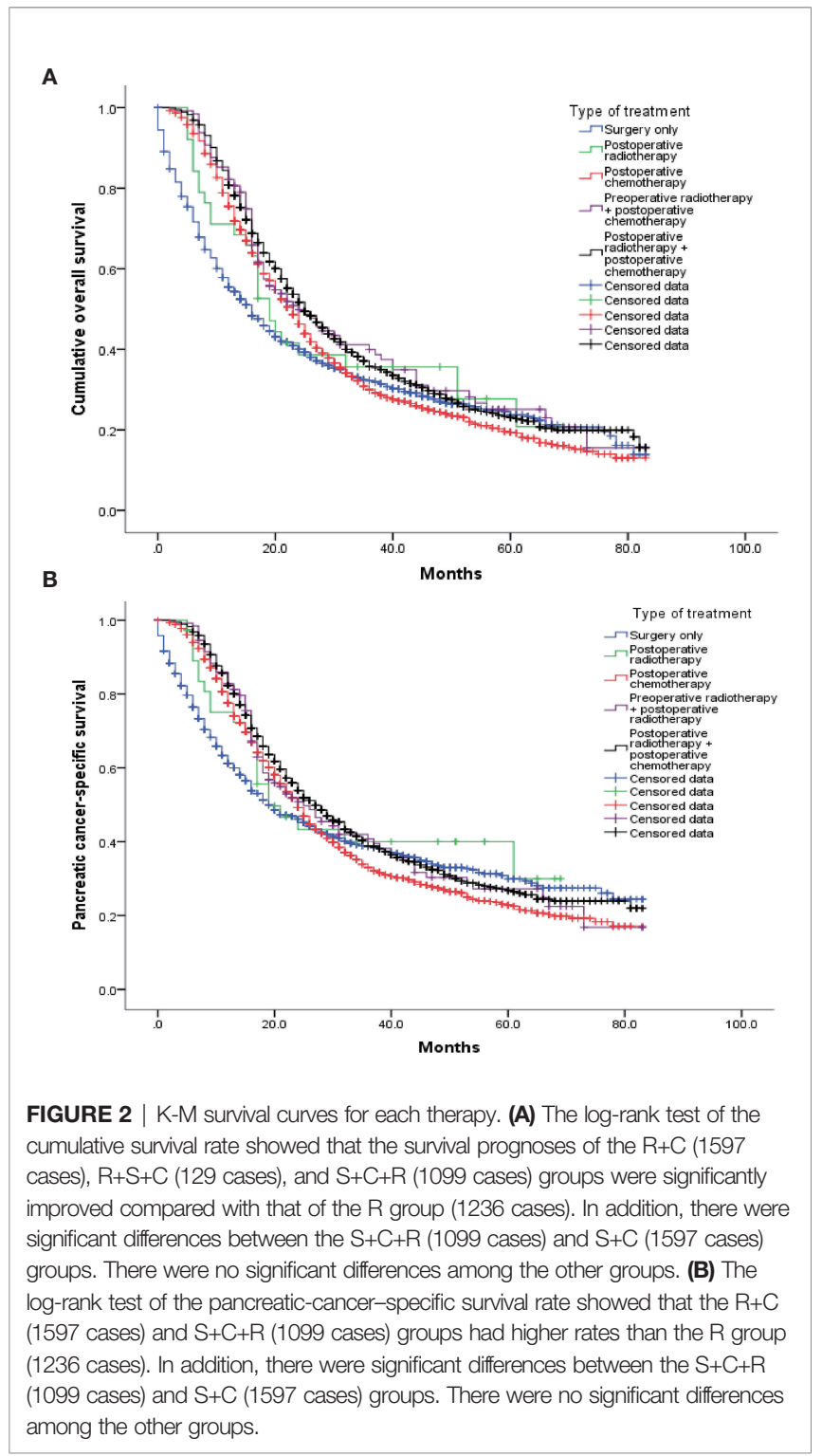

25 months and 16 months, respectively [HR $=0.736,95 \% \mathrm{CI}$ (0.658-0.822), $P<0.001$ ] (Figure 4).

\section{Construction of a Nomogram Prediction Model}

The results of the multivariate Cox regression model are shown in Table 2. The variables age, insurance status, gender, histology, degree of tissue differentiation, $\mathrm{T}$ and $\mathrm{N}$ stages, tumor size, regional lymph node dissection, and postoperative radiotherapy (Yes/No) or chemotherapy (Yes/No) were found to be statistically significant. The $\beta$ coefficient of the model was used to establish the nomogram (Figure 5). The model performance was calibrated and determined using the Bootstrap internal validation method. The calibration curves showed a good identity between the predicted and actual survival outcomes (Figure 6A). The area under the curve (AUC) was used to evaluate the discrimination of 1-year, 3 year, and 5-year overall survival with $0.740,0.743$, and 0.743 , respectively (Figure 6B).

\section{DISCUSSION}

In recent years, the incidences of carcinoma of the ampulla, distal cholangiocarcinoma, and pancreatic cancer (head, neck, and uncinate process carcinoma of pancreas) have gradually increased, and thus these cancers pose serious health risks. Pancreatoduodenectomy was developed more than 100 years ago and has evolved since then. It has been modified into many surgical procedures, including extended pancreatoduodenectomy and pylorus-preserving pancreatoduodenectomy. With the development of minimally invasive techniques and the clinical application of computers, pancreaticoduodenectomy can also be now performed using minimally invasive techniques, such as laparoscopy and robotics, and has been shown to be similar to the traditional open pancreaticoduodenectomy in terms of the outcome $(12,13)$. It should be noted that such minimally invasive procedures have the advantage of enhanced postoperative recovery. Although the prognosis of pancreaticoduodenectomy

TABLE 3 | Baseline characteristics of the patients in the postoperative-radiotherapy group before and after PSM.

\begin{tabular}{|c|c|c|c|c|c|c|}
\hline Variable & \multicolumn{2}{|c|}{ Before PSM } & $p$ & \multicolumn{2}{|c|}{ After PSM } & $p$ \\
\hline Insurance & & & 0.107 & & & 0.562 \\
\hline Medicaid & 93 & 304 & & 93 & 113 & \\
\hline Insured & 1027 & 2466 & & 1027 & 995 & \\
\hline Histology classification (ICD-O-3) & & & 0.266 & & & 0.997 \\
\hline Epithelial neoplasms & 8 & 33 & & 8 & 17 & \\
\hline Adenomas and adenocarcinomas & 566 & 1455 & & 566 & 559 & \\
\hline Cystic, mucinous and serous & 45 & 100 & & 45 & 36 & \\
\hline Ductal and lobular neoplasms & 511 & 1220 & & 511 & 513 & \\
\hline
\end{tabular}


TABLE 3 | Continued

\begin{tabular}{|c|c|c|c|c|c|c|}
\hline Variable & \multicolumn{2}{|c|}{ Before PSM } & $p$ & \multicolumn{2}{|c|}{ After PSM } & $p$ \\
\hline Complex epithelial neoplasms & 7 & 25 & & 7 & 12 & \\
\hline Grade I (well differentiated) & 120 & 494 & & 120 & 138 & \\
\hline Grade II (moderately differentiated) & 620 & 1333 & & 620 & 554 & \\
\hline Grade III (poorly differentiated) & 384 & 962 & & 384 & 424 & \\
\hline $\mathrm{T} 1$ & 35 & 212 & & 35 & 50 & \\
\hline T2 & 99 & 345 & & 99 & 78 & \\
\hline T3 & 955 & 2189 & & 955 & 968 & \\
\hline $\mathrm{T} 4$ & 48 & 87 & & 48 & 41 & \\
\hline $\mathrm{N}$ stage & & & $<0.001$ & & & 0.663 \\
\hline No & 279 & 981 & & 279 & 288 & \\
\hline Tumor size(mm) & $32.44 \pm 11.63$ & $32.62 \pm 15.03$ & 0.679 & $32.43 \pm 11.63$ & $32.40 \pm 12.98$ & 0.944 \\
\hline Chemotherapy (Yes/No) & & & $<0.001$ & & & 1 \\
\hline Yes & 38 & 1236 & & 38 & 38 & \\
\hline No & 1099 & 1597 & & 1099 & 1099 & \\
\hline
\end{tabular}

m, male; f, female; Scope Reg LN, Scope of regional Iymphadenectomy; ICD-O-3, The International Classification of Diseases for Oncology, Third Edition; PSM, Propensity score matching; Bold indicates a significant difference.

N, no; NA, not applicable no meta-analysis conducted; PY, partial yes; Y, yes.

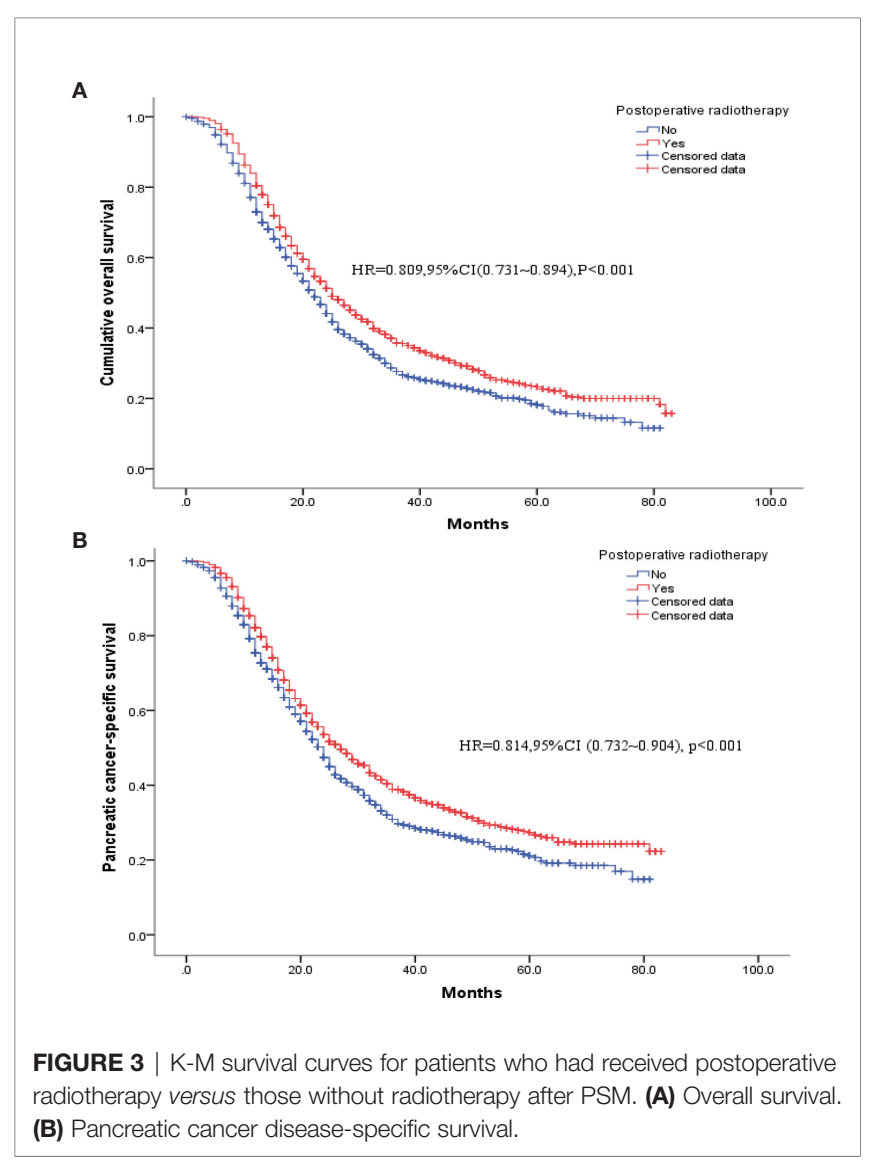

has improved by virtue of the technological developments and innovations in surgical techniques, this procedure is still one of the most technically complex and complicated procedures among abdominal surgical procedures. Identification of the factors affecting the prognosis of pancreatic cancer patients who have undergone pancreaticoduodenectomy will enable clinicians to customize an optimal treatment strategy.

In this study, we screened the data in the SEER database for patients diagnosed with pancreatic head cancer between 2010 and 2015 and selected the patients who had undergone pancreaticoduodenectomy. Based on the clinical variables provided by the SEER database, we found that age, insurance status, gender, histological type, degree of tissue differentiation, $\mathrm{T}$ and $\mathrm{N}$ stages, tumor size, extent of regional lymph node dissection, and postoperative radiotherapy or chemotherapy were independent factors affecting the prognosis of pancreaticoduodenectomy. We used this information to develop and validate a prognostic prediction nomogram. In addition, we also analyzed the effects of preoperative and postoperative adjuvant radiotherapies and postoperative adjuvant chemotherapy on postoperative survival. The results showed that postoperative radiotherapy or chemotherapy improves postoperative survival, whereas preoperative radiotherapy is of little benefit to patients. Because there are fewer patients receiving preoperative radiotherapy (only 129 cases) and these patients also received postoperative chemotherapy which may cause potential bias, the actual survival benefits of preoperative neoadjuvant radiotherapy still need to be further evaluated by more high-quality and better-designed studies in the future. 
TABLE 4 | Baseline characteristics of the patients in the postoperative-chemotherapy group before and after PSM.

\begin{tabular}{|c|c|c|c|c|c|c|}
\hline Variable & $\begin{array}{c}\text { Before PSM } \\
\text { Chemotherapy } \\
(n=2696)\end{array}$ & $\begin{array}{l}\text { No chemotherapy } \\
\qquad(n=1274)\end{array}$ & $p$ & $\begin{array}{c}\text { After PSM } \\
\text { Chemotherapy } \\
(n=1017)\end{array}$ & $\begin{array}{l}\text { No chemotherapy } \\
\qquad(n=1017)\end{array}$ & $p$ \\
\hline Age (year) & $65.15 \pm 10.01$ & $67.79 \pm 12.04$ & $<0.001$ & $66.90 \pm 9.97$ & $67.53 \pm 12.08$ & 0.193 \\
\hline $\operatorname{Sex}(m / f)$ & $1413 / 1283$ & $656 / 618$ & 0.588 & $513 / 504$ & $532 / 485$ & 0.399 \\
\hline Insurance & & & 0.009 & & & 0.606 \\
\hline Medicaid & 239 & 158 & & 97 & 114 & \\
\hline Insured & 2408 & 1085 & & 904 & 877 & \\
\hline Uninsured & 49 & 31 & & 16 & 26 & \\
\hline Race (White/Black/Other) & $2323 / 275 / 227$ & $1048 / 115 / 111$ & 0.98 & 856/83/78 & 856/83/78 & 1 \\
\hline Histology classification (ICD-O-3) & & & $<0.001$ & & & 0.81 \\
\hline epithelial neoplasms & 27 & 14 & & 16 & 11 & \\
\hline adenomas and & 1286 & 735 & & 517 & 526 & \\
\hline adenocarcinomas & & & & & & \\
\hline cystic, mucinous and serous & 91 & 54 & & 32 & 39 & \\
\hline ductal and lobular neoplasms & 1268 & 463 & & 442 & 433 & \\
\hline complex epithelial neoplasms & 24 & 8 & & 10 & 8 & \\
\hline Grade & & & $<0.001$ & & & 0.52 \\
\hline Grade I (well differentiated) & 296 & 318 & & 146 & 160 & \\
\hline Grade II (moderately differentiated) & 1369 & 584 & & 508 & 499 & \\
\hline Grade III (poorly differentiated) & 987 & 359 & & 344 & 345 & \\
\hline Grade IV (undifferentiated) & 44 & 13 & & 19 & 13 & \\
\hline T stage & & & $<0.001$ & & & 0.76 \\
\hline $\mathrm{T} 1$ & 114 & 133 & & 67 & 55 & \\
\hline $\mathrm{T} 2$ & 232 & 212 & & 107 & 135 & \\
\hline T3 & 2259 & 885 & & 809 & 785 & \\
\hline T4 & 91 & 44 & & 34 & 42 & \\
\hline $\mathrm{N}$ stage & & & $<0.001$ & & & 0.639 \\
\hline NO & 722 & 538 & & 337 & 347 & \\
\hline N1 & 1974 & 736 & & 680 & 670 & \\
\hline Scope Reg LN Sur & & & 0.025 & & & 0.902 \\
\hline None & 26 & 25 & & 13 & 11 & \\
\hline $1 \sim 3$ & 75 & 42 & & 26 & 27 & \\
\hline 4 or more & 2596 & 1207 & & 978 & 979 & \\
\hline Tumor size(mm) & $32.47 \pm 12.62$ & $32.77 \pm 16.90$ & 0.575 & $33.04 \pm 14.04$ & $32.60 \pm 14.19$ & 0.482 \\
\hline Radiotherapy (Yes/No) & & & $<0.001$ & & & 1 \\
\hline Yes & 1099 & 38 & & 38 & 38 & \\
\hline No & 1597 & 1236 & & 979 & 979 & \\
\hline
\end{tabular}

m, male; $f$, female; Scope Reg LN, Scope of regional lymphadenectomy; ICD-O-3, The International Classification of Diseases for Oncology, Third Edition; PSM, Propensity score matching; Bold indicates a significant difference.

From the analysis results, the prognosis of patients with pancreatic head cancer after pancreaticoduodenectomy is mainly closely related to the status of the tumor itself, including $\mathrm{T}$ and $\mathrm{N}$ stages, tumor size, histology, and degree of tissue differentiation. Specifically, late T or N stage, large tumor size, or low degree of tissue differentiation is correlated with poor prognosis. In addition, patient survival outcomes also differed by insurance status, age, and gender, but there was no significant difference in postoperative survival outcome among races. Specifically, patients with Medicare had better overall survival than those without Medicare or with basic Medicare. Old age was found to be associated with poor postoperative survival. This observation may be related to more comorbidities and poorer surgical tolerance in old patients than in young patients. Interestingly, the survival prognosis of the male patients was poorer than that of the female patients, and this observation is similar to the results of previous related studies as well as previous studies on other solid tumors (14-16).

With the development and promotion of neoadjuvant and adjuvant therapies, the current treatment strategy for pancreatic malignancies is a multidisciplinary and comprehensive treatment with surgery as the core. Especially, when endoscopic ultrasoundguided fine-needle aspiration (EUS-FNA) can be used for the diagnosis of solid pancreatic lesions $(17,18)$, a large number of pancreatic cancers can be pathologically diagnosed before surgery. This has prompted some experts to think about whether postoperative adjuvant therapy can be treated in advance, that is, neoadjuvant therapy. But the pro-fibrotic response and cytotoxicity induced by neoadjuvant chemotherapy causes loss of healthy tissue planes and poses a challenge to any surgical procedure. However, the SEER database lacks records of preoperative chemotherapy for pancreatic cancer, preventing us from obtaining data on patients who had received neoadjuvant chemotherapy. The SEER database has only the records of pancreatic cancer patients who had received postoperative chemotherapy. In recent years, FOLFIRINOX (Fluorouracil + Leucovorin + Irinotecan + Oxaliplatin) and NabPaclitaxel Plus Gemcitabine have been shown to result in significant improvements in the survival of patients with pancreatic malignancies (19). In this study, approximately 69\% (2825/4099) of the patients in our study had been recorded in the database to have 


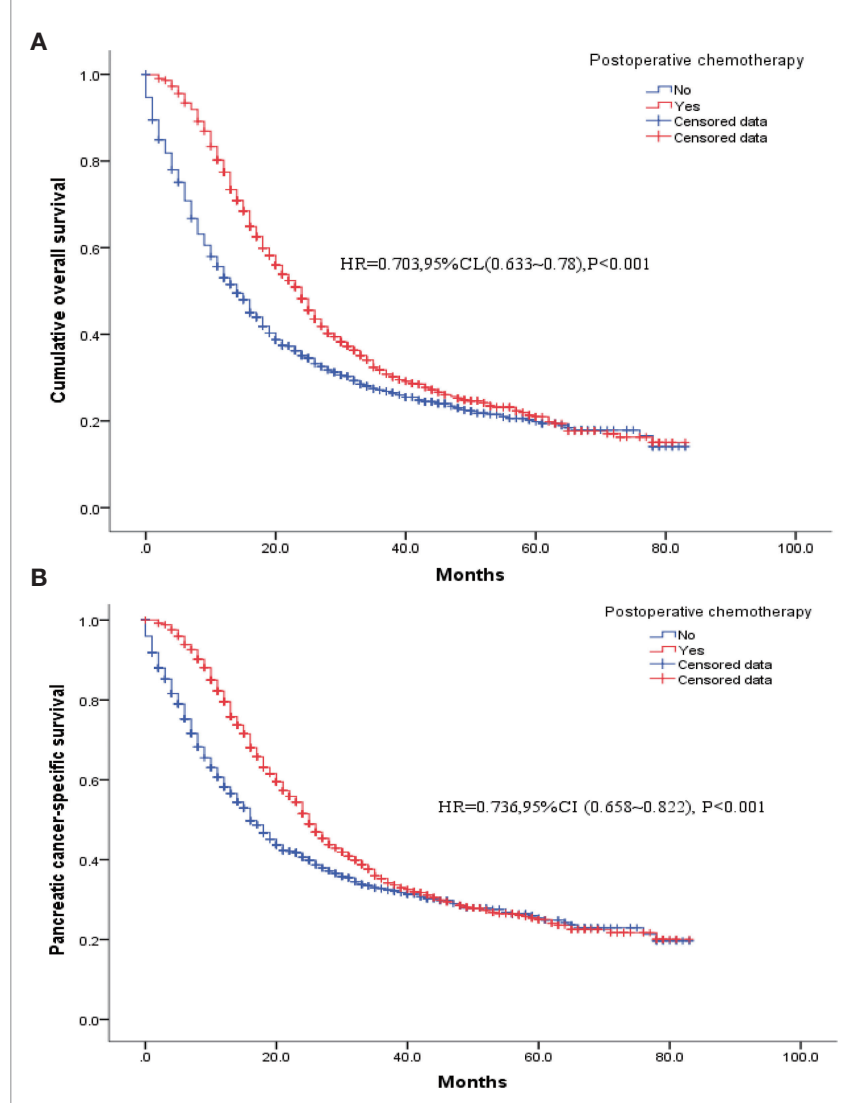

FIGURE 4 | K-M survival curves for patients who had received postoperative chemotherapy versus patients without chemotherapy after PSM. (A) Overall survival. (B) Pancreatic cancer disease-specific survival.

received postoperative chemotherapy, resulting in a significantly longer survival time of 8 months ( $\mathrm{HR}=0.773$, 95\% CI: $0.713-$ 0.838). Of the total 4099 patients, 1266 patients (30.9\%) had received radiation therapy. The proportion receiving postoperative radiation was significantly higher in patients receiving postoperative chemotherapy than in those not receiving, and postoperative radiation therapy may have biased the effect of chemotherapy; therefore, we put unbalanced variables, including radiation therapy data, into covariates for PSM to obtain newly balanced data (1017 patients receiving postoperative chemotherapy and 1017 patients not receiving postoperative chemotherapy). The new data analysis showed that the patients who had received postoperative chemotherapy continued to have higher survival rates than those who had not received postoperative chemotherapy, with their median survivals being 24 and 14 months, respectively [HR $=0.703,95 \% \mathrm{CI}$ (0.633-0.78), $\mathrm{P}<0.001]$, and the pancreatic-cancer-specific survivals being 25 and 16 months, respectively [HR $=0.736,95 \%$ CI $(0.658-$ 0.822), $\mathrm{P}<0.001]$.

At present, this study is one of the largest studies in the real world evaluating the effects of radiation therapy in pancreatic cancer patients undergoing pancreaticoduodenectomy. In our study, 129 (3.14\%) of the 4099 patients received preoperative radiotherapy and 1137 patients $(27.7 \%)$ received postoperative radiotherapy. Both univariate and multifactorial Cox regression analyses found that postoperative radiotherapy was beneficial for the patients, unlike preoperative radiotherapy. Similarly, the proportion of patients receiving postoperative chemotherapy was significantly higher in patients who had received radiotherapy than in those who had not; therefore, PSM was performed to obtain balanced cohort data (1137 patients who had received postoperative radiotherapy and 1137 who did not). The results from the new analysis showed that the prognosis of the patients who had received postoperative radiotherapy was better than those who had not, and their median postoperative survivals were 25 and 22 months, respectively [HR $=0.809$, 95\% CI $(0.731-$ $0.894), \mathrm{P}<0.001]$, and the pancreatic-cancer-specific median survivals were 24 and 27 months, respectively [HR $=0.814,95 \%$ CI (0.732-0.904), P < 0.001].

Radiation technology has greatly improved, and over time, radiotherapy techniques will be more precise in delivering the maximum dose to the tumor target and the minimum dose to the healthy tissue. In addition, any shift in the location of the pancreatic tumor due to respiration is taken into account via imaging comparisons and integration before each treatment (20). As reported by Wang et al, receiving both radiotherapy and chemotherapy was found to significantly improve the overall survival in locally advanced and metastatic pancreatic cancer within an acceptable toxicity range. A similar conclusion was obtained in the present study that patients undergoing pancreaticoduodenectomy with both radiotherapy and chemotherapy after surgery could achieve a greater benefit than those with monotherapy (Figure 5).

The nomogram developed in this study overcomes the shortcomings, including low specificity as well as low accuracy, of the AJCC TNM system and a previously developed nomogram $(9,21)$ for predicting the postoperative survival in pancreatic head cancer. When patients were predicted for survival by this nomogram, other variables, including gender, age, insurance status, tumor histological classification, degree of differentiation, extent of regional lymph node dissection, and postoperative radiotherapy or chemotherapy, together with the degree of tumor invasion, tumor size, and lymph node metastasis, were identified as prognostic risk factors. This nomogram was specially designed for patients with pancreatic head cancer who have undergone or will undergo pancreaticoduodenectomy. The results of the Bootstrap internal validation method showed that the predicted and observed values were similar for 1-, 3-, and 5year survival rates (Figure 6A). From a practical point of view, the variables incorporated in the nomogram are also readily available in patients undergoing pancreaticoduodenectomy. With this simple and easily available information, clinicians can accurately predict survival and provide patients with valid information about different treatment options.

The merit of this study is that the conclusions were derived from the large population and authoritative data in the SEER database, whereby the risks of selection and publication biases were minimized. Since the SEER data had been derived from a large number of unselected patients, the conclusions of this study are universally applicable to populations, such as those in other 


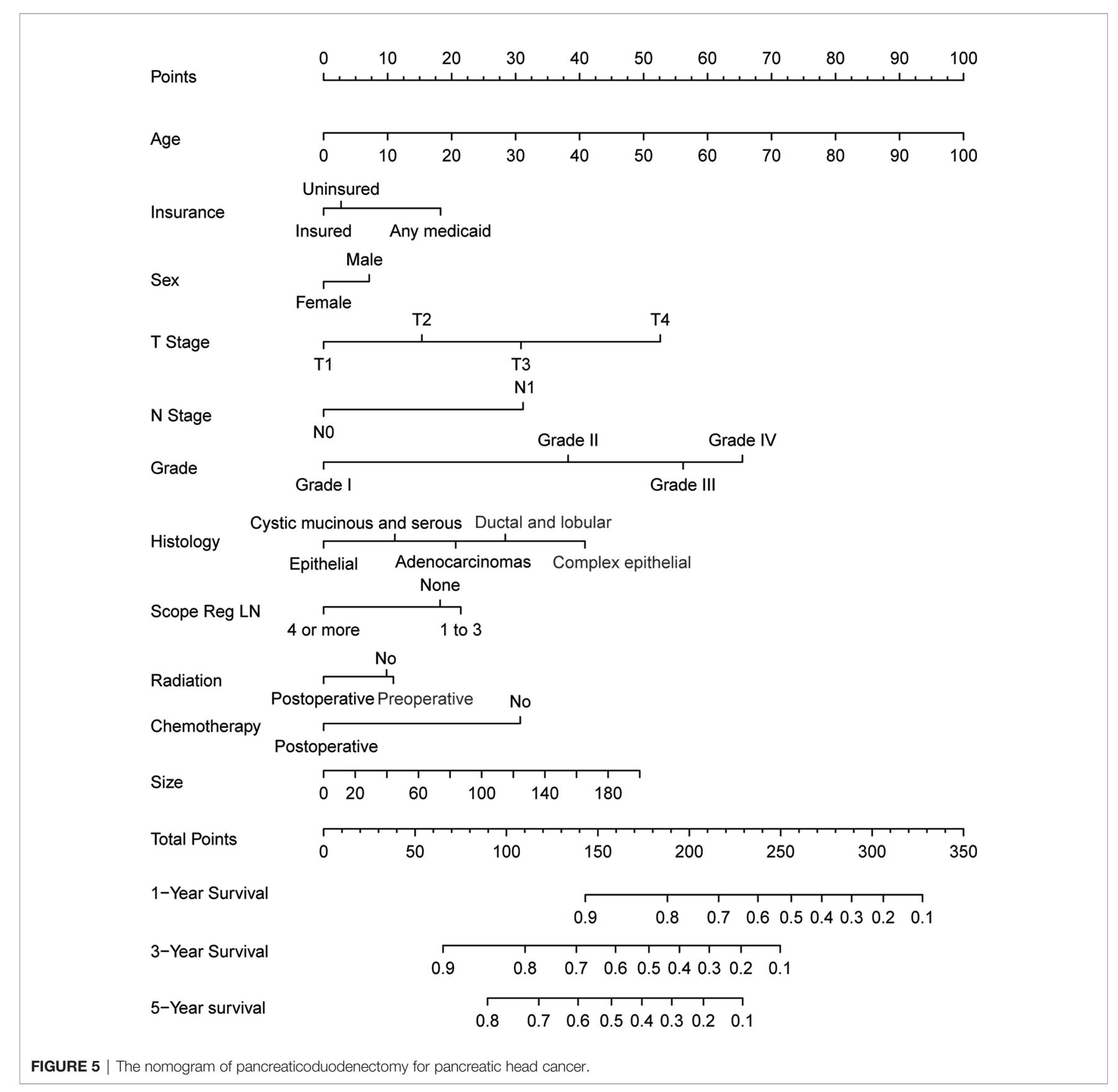

regions of the world. In this study, after analyzing the factors affecting postoperative survival, a new prediction model was established. This prognostic prediction model may guide clinicians in the treatment of pancreatic cancer and enable them to customize effective treatment strategies.

\section{Limitations}

Undeniably, there are several limitations in our analysis. First of all, some important laboratory data (such as CA19-9, CA125, creactive protein, neutrophil-to-lymphocyte ratio) and other factors that may affect the prognosis were not included in our analysis because of the lack of corresponding records in the SEER database. Although we used PSM to reduce bias, the residual confusion due to the unrecorded variables in the SEER database cannot be disregarded. Therefore, some potentially unknown influencing factors may bias the analysis results. Nevertheless, the SEER database provides sufficient sample volume to reduce possible bias, and our conclusions are still of great informative value. Second, due to the absence of neoadjuvant chemotherapy data and insufficient neoadjuvant radiotherapy data in the SEER database, we cannot accurately assess the effect of neoadjuvant 

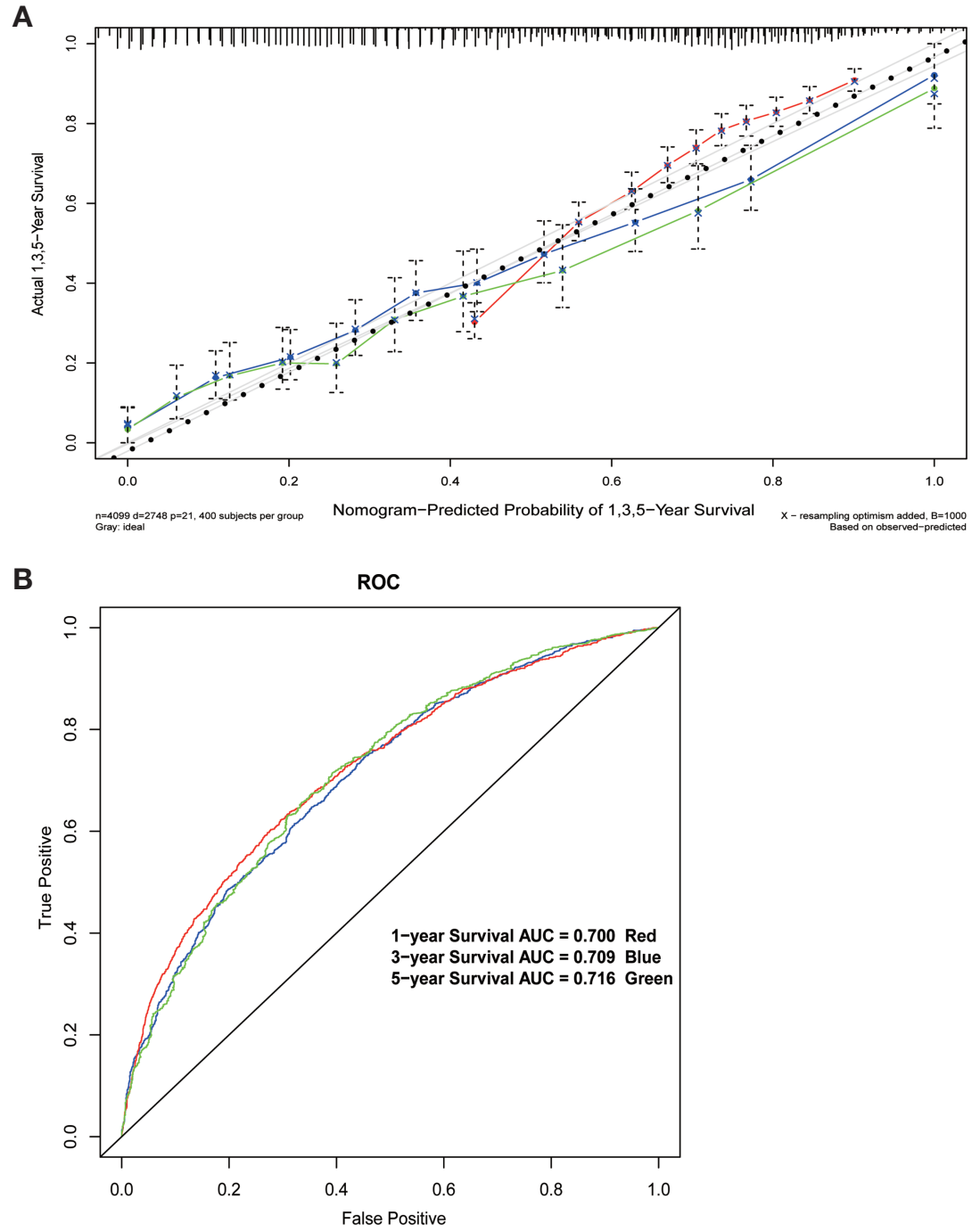

FIGURE 6 | (A) Calibration curve of the nomogram. (B) 1-year, 3-year, and 5-year Receiver Operating Characteristic curves of the nomogram.

radiotherapy and chemotherapy before surgery. Also, the long enrollment period of patients may also be a limitation, because the changes and improvements in surgery, chemotherapy, and radiotherapy techniques can bias the conclusions. Finally, we used the Bootstrap internal validation method to evaluate the model performance. The results showed that our prediction model has a good performance, but we do not know the accuracy of the model application since the model lacks external validation based on other cohorts.

\section{CONCLUSION}

Age at diagnosis, medical insurance, gender, pathological tissue type and degree of differentiation, $\mathrm{T}$ and $\mathrm{N}$ stages of the tumor, tumor size, the intraoperative extent of lymph node dissection, and postoperative radiotherapy or chemotherapy are independent prognostic factors for pancreatic head cancer patients undergoing pancreaticoduodenectomy. Specifically, when patients are old, without health insurance, male, with pathological tissues in the order of epithelial, cystic mucinous and plasmacytic tumors, adenomas, ductal and lobular tumors, and complex epithelium, low degree of tissue differentiation, advanced $\mathrm{T}$ or $\mathrm{N}$ stage, or low regional lymphadenectomy is correlated with poor prognosis for postoperative survival. Postoperative radiotherapy or chemotherapy significantly improves patient survival. These conclusions should be verified through high-quality prospective studies in the future. The nomogram prediction model developed in this paper has a good prediction accuracy and can enable clinicians to predict the survival of a patient with pancreatic head cancer after pancreaticoduodenectomy. 


\section{DATA AVAILABILITY STATEMENT}

The raw data supporting the conclusions of this article will be made available by the authors, without undue reservation.

\section{AUTHOR CONTRIBUTIONS}

WZ was responsible for drafting the manuscript, as well as the acquisition, analysis and interpretation of data. LX and XC

\section{REFERENCES}

1. Siegel RL, Miller KD, Jemal A. Cancer Statistics, 2017. CA Cancer J Clin (2017) 67(1):7-30. doi: 10.3322/caac.21387

2. He Y, Liang D, Li D, Shan B, Zheng R, Zhang S, et al. Incidence and Mortality of Laryngeal Cancer in China, 2015. Chin J Cancer Res (2020) 32(1):10-7. doi: 10.21147/j.issn.1000-9604.2020.01.02

3. Whipple AO, Parsons WB, Mullins CR. Treatment of Carcinoma of The Ampulla of VATER. Ann Surg (1935) 102(4):763-79. doi: 10.1097/00000658193510000-00023

4. Iasonos A, Schrag D, Raj GV, Panageas KS. How to Build and Interpret a Nomogram for Cancer Prognosis. J Clin Oncol (2008) 26(8):1364-70. doi: 10.1200/JCO.2007.12.9791

5. Kong X, Li J, Cai Y, Tian Y, Chi S, Tong D, et al. A Modified TNM Staging System for Non-Metastatic Colorectal Cancer Based on Nomogram Analysis of SEER Database. BMC Cancer (2018) 18(1):50. doi: 10.1186/s12885-0173796-1

6. Albert JM, Liu DD, Shen Y, Pan IW, Shih YC, Hoffman KE, et al. Nomogram to Predict the Benefit of Radiation for Older Patients With Breast Cancer Treated With Conservative Surgery. J Clin Oncol (2012) 30(23):2837-43. doi: 10.1200/JCO.2011.41.0076

7. Wang Y, Li J, Xia Y, Gong R, Wang K, Yan Z, et al. Prognostic Nomogram for Intrahepatic Cholangiocarcinoma After Partial Hepatectomy. J Clin Oncol (2013) 31(9):1188-95. doi: 10.1200/JCO.2012.41.5984

8. Are C, Afuh C, Ravipati L, Sasson A, Ullrich F, Smith L. Preoperative Nomogram to Predict Risk of Perioperative Mortality Following Pancreatic Resections for Malignancy. J Gastrointest Surg (2009) 13(12):2152-62. doi: 10.1007/s11605-009-1051-Z

9. Pan J, Hou Y, Zhang D, Wu H, Liu X, Liu J, et al. Efficacy and Prognostic Factors of Pancreaticoduodenectomy for Duodenal Papillary Carcinoma. J Hepatobil Pancreatic Surg (2012) 24(05):362-6. doi: 10.1007/s00330-02006864-Z

10. Zhu S, Zhu M, Wang Z, Chen Y, et al. Multivariate Analysis of Pancreatic Cancer and Pancreaticoduodenectomy to Assess Prognosis. International Hepatobiliary and Pancreatic Surgery Nantong Forum and National Hepatobiliary and Pancreatic Surgery Academic Experience Exchange Conference. Chinese Medical Association; Jiangsu Medical Association (2009).

11. Allen PJ, Kuk D, Castillo CF, Basturk O, Wolfgang CL, Cameron JL, et al. MultiInstitutional Validation Study of the American Joint Commission on Cancer (8th Edition) Changes for T and N Staging in Patients With Pancreatic Adenocarcinoma. Ann Surg (2017) 265(1):185-91. doi: 10.1097/SLA.0000000000001763

12. Zhang W, Huang Z, Zhang J, Che X. Safety and Efficacy of Robot-Assisted Versus Open Pancreaticoduodenectomy: A Meta-Analysis of Multiple Worldwide Centers. Updates Surg (2021) 73(3):893-907. doi: 10.1007/s13304-020-00912-5

13. Lyu Y, Cheng Y, Wang B, Xu Y, Du W. Minimally Invasive Versus Open Pancreaticoduodenectomy: An Up-To-Date Meta-Analysis of Comparative Cohort Studies. J Laparoendosc Adv Surg Tech A (2019) 29(4):449-57. doi: 10.1089/lap.2018.0460 contributed to the conception and design of the current study. All authors contributed to the article and approved the submitted version.

\section{FUNDING}

This research supported by Sanming Project of Medicine in Shenzhen (No. SZSM 201911008 and SZSM 202011010).

14. Amin S, Lucas AL, Frucht H. Evidence for Treatment and Survival Disparities by Age in Pancreatic Adenocarcinoma: A Population-Based Analysis. Pancreas (2013) 42(2):249-53. doi: 10.1097/MPA.0b013e31825f3af4

15. Osawa M, Akuta N, Suzuki F, Fujiyama S, Kawamura Y, Sezaki H, et al. Prognosis and Predictors of Hepatocellular Carcinoma in Elderly Patients Infected With Hepatitis B Virus. J Med Virol (2017) 89(12):2144-8. doi: $10.1002 /$ jmv. 24890

16. Kula D, Kalemba M, Puch Z, Polańska J, Swierniak M, Rusinek D, et al. Age at Diagnosis and Gender Modify the Risk of 9q22 and 14q13 Polymorphisms for Papillary Thyroid Carcinoma. Endokrynol Pol (2017) 68(3):283-9. doi: 10.5603/EP.2017.0021

17. Crinò SF, Ammendola S, Meneghetti A, Bernardoni L, Conti Bellocchi MC, Gabbrielli A, et al. Comparison Between EUS-Guided Fine-Needle Aspiration Cytology and EUS-Guided Fine-Needle Biopsy Histology for the Evaluation of Pancreatic Neuroendocrine Tumors. Pancreatology (2021) 21(2):443-50. doi: 10.1016/j.pan.2020.12.015

18. Crinò SF, Larghi A, Bernardoni L, Parisi A, Frulloni L, Gabbrielli A, et al. Touch Imprint Cytology on Endoscopic Ultrasound Fine-Needle Biopsy Provides Comparable Sample Quality and Diagnostic Yield to Standard Endoscopic Ultrasound Fine-Needle Aspiration Specimens in the Evaluation of Solid Pancreatic Lesions. Cytopathology (2019) 30(2):179-86. doi: 10.1111/cyt.12662

19. Sohal DP, Mangu PB, Laheru D. Metastatic Pancreatic Cancer: American Society of Clinical Oncology Clinical Practice Guideline Summary. J Oncol Pract (2017) 13(4):261-4. doi: 10.1200/JOP.2016.017368

20. Wang Z, Ren ZG, Ma NY, Zhao JD, Zhang Z, Ma XJ, et al. Intensity Modulated Radiotherapy for Locally Advanced and Metastatic Pancreatic Cancer: A Mono-Institutional Retrospective Analysis. Radiat Oncol (2015) 10:14. doi: 10.1186/s13014-014-0312-5

21. Li HB, Zhou J, Zhao FQ. A Prognostic Nomogram for Disease-Specific Survival in Patients With Pancreatic Ductal Adenocarcinoma of the Head of the Pancreas Following Pancreaticoduodenectomy. Med Sci Monit (2018) 24:6313-21. doi: 10.12659/MSM.909649

Conflict of Interest: The authors declare that the research was conducted in the absence of any commercial or financial relationships that could be construed as a potential conflict of interest.

Publisher's Note: All claims expressed in this article are solely those of the authors and do not necessarily represent those of their affiliated organizations, or those of the publisher, the editors and the reviewers. Any product that may be evaluated in this article, or claim that may be made by its manufacturer, is not guaranteed or endorsed by the publisher.

Copyright (c) 2021 Zhang, Xu and Che. This is an open-access article distributed under the terms of the Creative Commons Attribution License (CC BY). The use, distribution or reproduction in other forums is permitted, provided the original author(s) and the copyright owner(s) are credited and that the original publication in this journal is cited, in accordance with accepted academic practice. No use, distribution or reproduction is permitted which does not comply with these terms. 